\title{
A Review of the Imaging Techniques for Measuring Kidney and Cyst Volume in Establishing Autosomal Dominant Polycystic Kidney Disease Progression
}

\author{
Riccardo Magistroni ${ }^{a} \quad$ Cristiana Corsi $^{b}$ Teresa Martíc, e Roser Torra ${ }^{d}$ \\ aSurgical, Medical and Dental Department of Morphological Sciences related to Transplant, Oncology and

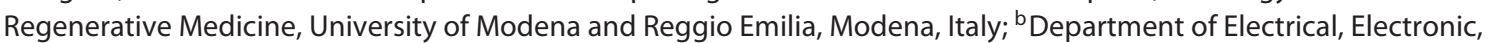 \\ and Information Engineering "Guglielmo Marconi," University of Bologna, Cesena, Italy; ' Department of Radiology, \\ Fundació Puigvert, Barcelona, Spain; ' Department of Nephrology, Inherited Renal Disorders, Fundació Puigvert, \\ REDINREN, IIB Sant Pau, Universitat Autónoma de Barcelona, Barcelona, Spain; ' Department of Radiology, \\ Fundació Hospital Esperit Sant, Barcelona, Spain
}

\section{Keywords}

Autosomal dominant polycystic kidney disease .

Computed tomography · Magnetic resonance imaging ·

Renal · Ultrasound

\begin{abstract}
Background: Autosomal dominant polycystic kidney disease (ADPKD) is the commonest inherited renal disorder; it is defined by progressive renal cyst formation and subsequent renal enlargement that leads to end-stage renal disease. Until recently, only symptomatic treatments for ADPKD existed. However, therapies that address the underlying pathophysiology of ADPKD are now available and accurate identification of the rate of disease progression is essential. Summary: Published data on the different imaging modalities for measuring kidney and cyst volumes in ADPKD are reviewed. The advantages and drawbacks of the different techniques for calculating kidney volume from renal imaging are also examined, including the use of manual planimetry, stereology, and the ellipsoid equation, as well as the
\end{abstract}

(C) 2018 S. Karger AG, Basel

\section{E-Mail karger@karger.com www.karger.com/ajn \\ KARGER}

prospect of semi- and fully automatic techniques. The translation of these approaches into clinical practice and their role in informing treatment decisions is discussed. Key Messages: These new therapies require the accurate monitoring of disease progression, which along with diagnosis and prognosis, relies on the effective use of renal imaging techniques. There is growing support for the use of total kidney volume as a measure of cyst burden and as a prognostic predictor of renal function in ADPKD, showing promise as a marker of disease progression.

(c) 2018 S. Karger AG, Basel

\section{Introduction}

Autosomal dominant polycystic kidney disease (ADPKD) is the commonest inherited renal disorder and it accounts for approximately $10 \%$ of all patients with end-stage renal disease (ESRD) in Europe [1]. ADPKD is characterized by the progressive development of fluid-filled renal cysts originating in $1-2 \%$ of nephrons 
[2]. Progressive cyst formation causes kidney enlargement, resulting in signs and symptoms that include pain, hypertension, hematuria, cyst and urinary tract infections, and renal failure [3]. Although cysts develop from birth, due to compensatory hyperfiltration in non-cystic tubules, renal function decline is not usually apparent until the fourth or fifth decade of life, depending on the mutation present [4].

ADPKD is caused by mutations in PKD1 or PKD2 genes, accounting for approximately 85 and $15 \%$ of cases, respectively [5], and de novo mutations can occur [6]. Patients with PKD1 mutations, particularly truncating mutations, present with more severe form of the disease than those with PKD2 mutations, and the median age at the onset of ESRD is approximately 58 and 79 years, respectively [7].

Until recently, treatments for ADPKD were symptomatic, without affecting cyst formation and consequent renal enlargement [8]. However, in 2012, in the 3-year TEMPO 3:4 study of 1,445 patients with ADPKD, the vasopressin $\mathrm{V}_{2}$ receptor antagonist tolvaptan was shown to significantly reduce the annual increase in total kidney volume (TKV) [9]. In 2015, the European Medicines Agency (EMA) granted approval for tolvaptan to be used to slow disease progression in ADPKD, in adults with chronic kidney disease stages 1-3 at initiation of treatment in the presence of evidence of rapidly progressing disease [10]. In the United States, the Food and Drug Administration approved tolvaptan in 2018 to be used to slow kidney function decline in adults at risk of rapidly progressing ADPKD [11].

TKV is an important measure for assessing disease progression, as it can determine prognosis through its ability to predict decline in renal function [8]. TKV continuously increases by an average of $5-6 \%$ per year throughout the course of ADPKD [9, 12], irrespective of the mutation present. Increase in kidney size is driven by an exponential increase in cyst volume as well as cyst number and initiation rate [13].

It has been suggested that changes in TKV (or TKV adjusted for height and age) can identify patients at risk of rapid progression to ESRD $[8,14-17]$ and can consequently enrich clinical trials. Recent studies have used changes in TKV as a primary endpoint $[9,12]$. The role of TKV in evaluating ADPKD progression and its possible role as a surrogate endpoint in randomized clinical trials has also been reviewed [18]. However, some studies have indicated a lack of correlation between TKV growth rate and decline in renal function $[19,20]$, leading to discussions regarding the use of TKV as a secondary end- point. The Polycystic Kidney Disease Outcomes Consortium recently established a therapeutic data standard for ADPKD [21]. This allowed for observational data from multiple sources, including the Consortium for Radiologic Imaging Studies of Polycystic Kidney Disease (CRISP) [21], to be integrated into a single data set that included $\leq 30$ years of follow-up TKV data from 2,355 patients with ADPKD [22]. Analysis of this dataset showed that baseline TKV had a significant impact on the risk of a 30\% decline in estimated glomerular filtration rate (eGFR) and progression to ESRD, independent of baseline eGFR and age [22]. The recent CRISP III study utilized 14.5 years of follow-up data in patients with ADPKD and showed that baseline TKV adjusted for height (ht T$\mathrm{KV}$ ) was an independent predictor of $\mathrm{a} \geq 30$ and $\geq 57 \%$ decline in eGFR from baseline, with a significant correlation between the annual rate of htTKV increase and eGFR decline [23].

The increasing use of TKV as a marker of ADPKD progression raises the question of how best to measure TKV and changes in it. This review discusses the rationale for using TKV in ADPKD in both progression models and clinical practice, and also gives information on the different imaging techniques available for measuring kidney and/or cyst volume in clinical practice.

\section{Imaging in Clinical Practice}

After the EMA approved tolvaptan for patients with rapid disease progression, the European Renal Association-European Dialysis and Transplant Association (ERA-EDTA) Working Groups on Inherited Kidney Disorders and European Renal Best Practice (WGIKD/ ERBP) published a position statement aimed at homogenizing the criteria for the definition of a patient with rapid ADPKD progression. The statement's recommendations included an algorithm to assess indications for initiation of ADPKD treatment. The algorithm requires predicted progression by baseline htTKV where data on eGFR decline are unavailable or unreliable, as well as the use of historical TKV data [14].

According to our experience, ultrasound examination could constitute the initial assessment for preliminary stratification of patients according to the risk of ADPKD progression. Considering the low accuracy and reproducibility of ultrasound, this estimation should be considered conclusive only for patients with near-normal kidney volumes. For dimensional increases in kidney size, an initial magnetic resonance imaging (MRI) scan is advised. How-
68

Am J Nephrol 2018;48:67-78 DOI: $10.1159 / 000491022$
Magistroni/Corsi/Martí/Torra 
ever, ultrasound may be useful in identifying young $\mathrm{AD}$ PKD patients with massively enlarged kidneys for their age and height; in these cases, an MRI scan may not be mandatory but is still recommended [14].

The inaccuracies associated with ultrasound, and therefore the potential for misclassification of the risk of progression, limit its application in clinical practice when a clinical decision regarding initiation of therapy is required. Furthermore, when high sensitivity is required, the efficiency and accuracy of semiautomated and automated approaches for volume estimation are recommended over methods based on geometric assumption (e.g., ellipsoid or mid-slice approaches). However, where access to MRI or computed tomography (CT) for TKV evaluation is limited, the ellipsoid formula by ultrasound could be a viable alternative.

Where available, three-dimensional imaging techniques are preferred because they offer more precise and reproducible estimates of TKV, although their limitations should be considered. While reformatted images may be required to identify the largest kidney diameters, this increases interobserver variability. This adds to the complexity of measuring disease progression over time and requires the accurate recording of reformatted kidney diameters to reproduce the images.

A single bulging cyst in the periphery of a kidney can elevate kidney diameter measurements, subsequently causing difficulty in obtaining accurate estimates of TKV. Guidance is not available on whether to include or discount bulging cysts, and the decision must be made in clinical practice. Obtaining kidney contours from which three-dimensional patient-specific kidney surfaces can be visualized may enable the identification of a bulging cyst, as well as the number and volume of cysts. This information could be integrated with other imaging modalities such as diffusion-weighted and diffusion-tensor imaging.

Overall, the most adequate method for TKV computation depends on several issues, including the availability of different imaging systems in clinical centers and the accuracy required by the clinical question. In a situation in which the required technology is available and the clinical question requires high accuracy, an MRI scan using an automated or semiautomated approach is accurate and convenient. Any other manual method or method based on geometrical approximation is not recommended because of the accuracy-to-cost ratio compared with that for the semiautomated and automated approaches. Volumetric follow-up may be necessary after an initial MRI scan when, for example, a patient is categorized as class $1 \mathrm{~A}$ by the Mayo Clinic model for disease progression and there is a risk of shifting between classes during follow-up [17]. In this case, follow-up evaluations could be based on an ultrasound examination applying the ellipsoid method. However, if the patient falls into classes $1 \mathrm{~B}$ or $1 \mathrm{C}$, volume quantification by MRI, using an automated or semiautomated method would be preferable for an accurate assessment of risk of progression.

Considering the small changes in kidney volume seen at patient follow-up, volumetric evaluation should not be performed more frequently than every 12 months unless clinically justified. Ideally, for a comprehensive evaluation, our suggestion is to perform an initial three-dimensional MRI or CT scan in order to have a reliable baseline radiological evaluation of the kidneys. Successive monitoring could be performed using ultrasound, applying geometrical models for volume computation. If such monitoring highlights an increase in kidney size or an irregular shape that makes ultrasound - and therefore the related methods for volume estimates - unreliable, an MRI should be performed.

In view of the estimation error, the only approach advisable for volumetric follow-up of kidney enlargement is the semiautomated or automated method from an MRI or CT scan. However, in clinical practice, TKV follow-up has not been recommended for the assessment of treatment efficacy. Clinical trials have used TKV follow-up from baseline as a measure of treatment response in patients with ADPKD; however, these results have traditionally been based on the average TKV change in large patient cohorts. When considering the individual patient, the measurement error intrinsic to imaging methodology makes it difficult to discern the effect of treatment from the background noise. For this reason, serial TKV followup in individual patients is not usually performed in routine clinical practice for assessing treatment response, as the difficulty in interpreting the results would be unlikely to lead to changes in therapeutic approach.

\section{Imaging Modalities}

TKV can be measured by ultrasound, MRI, or CT using manual, semiautomated, or fully automated data processing techniques (Table 1). Imaging examples of the manual TKV estimation methods are shown in Figure 1A.

\section{Ultrasound}

Ultrasound is important in prognostic disease stratification in clinical practice. A position statement from the ERA-EDTA WGIKD/ERBP sought to define ADPKD pa- 
Table 1. Summary of ADPKD imaging methodologies for measuring kidney and cyst volumes

ADPKD imaging modalities

Ultrasound

Measurement accuracy

- Can detect cysts $>1 \mathrm{~cm}$ in diameter, although 7-10 $\mathrm{mm}$ is the smallest size that can be detected with confidence [52]; new-generation scanners can detect cysts that are 2-3 $\mathrm{mm}$ in diameter [55]

- Coefficients of variation for kidney volume measurements using geometric modeling shown to be $18-42 \%$, largely due to interoperator variability [39]

\begin{tabular}{|c|c|}
\hline Advantages & $\begin{array}{l}\text { - Does not require radiation or contrast material } \\
\text { - Widely available } \\
\text { - Low cost } \\
\text { - Safety } \\
\text { - Well established diagnostic criteria }[8,55]\end{array}$ \\
\hline Drawbacks & - Lacks precision and accuracy for detecting short-term changes in kidney volume \\
\hline $\begin{array}{l}\text { MRI } \\
\text { Measurement accuracy }\end{array}$ & $\begin{array}{l}\text { - Can detect cysts } \geq 2 \mathrm{~mm} \text { in diameter [52] } \\
\text { - Coefficients of variation for kidney volume measurements using geometric modeling shown to be } \\
1.7 \%[39]\end{array}$ \\
\hline Advantages & $\begin{array}{l}\text { - Provides high resolution and tissue contrast three-dimensional images and does not use radiation or } \\
\text { iodinated contrast medium }[25] \\
\text { - Can reliably measure kidney volume over short periods of time with minimal bias and low } \\
\text { inter- and intraoperator variability }[15,25,26] \\
\text { - Allows segmentation of individual cysts providing quantitative assessment of disease severity in } \\
\text { patients with early or moderate ADPKD [45] }\end{array}$ \\
\hline Drawbacks & $\begin{array}{l}\text { - Cost } \\
\text { - Lack of availability } \\
\text { - Variability in imaging results between scanners } \\
\text { - Time needed for image acquisition (up to } 12 \text { min for } \mathrm{T}_{2} \text {-weighted images) [30] } \\
\text { - Patient-related factors (claustrophobia, metallic medical implants) }\end{array}$ \\
\hline
\end{tabular}

CT

Measurement accuracy - Can detect cysts $\geq 2 \mathrm{~mm}$ in diameter [52]

Advantages _ - Provides accurate and reliable measurements of TKV and cyst volume in ADPKD [34, 56], which correlate well with values obtained by ultrasound [41]

Drawbacks - Potentially nephrotoxic contrast medium

- Exposure of patient to ionizing radiation

Kidney volume analysis techniques*

\begin{tabular}{llll}
\hline Imaging modality & Volume analysis & Analysis time, min & $\begin{array}{l}\text { Measurement accuracy according to Mayo } \\
\text { Clinic model classification, \% }\end{array}$ \\
\hline \multirow{2}{*}{ MRI $\left(\mathrm{CT}^{\dagger}\right)$} & Manual planimetry & 40 & 100 (gold standard) \\
& Stereology & N/A & 57 \\
& Ellipsoid formula & 5 & 90 \\
Mid-slice & 7 & 97.5 \\
& Semiautomated & 2 & N/A \\
& Fully automated & N/A & 21 \\
\hline Ultrasound & Ellipsoid formula & 5 & \\
\hline
\end{tabular}

* Data on kidney volume analysis techniques adapted from Turco et al. [57]; the accuracy of each volume analysis method was evaluated according to the correlation of each method with Mayo Clinic model classifications performed according to the MRI/manual method, which is considered to be the gold standard; ${ }^{\dagger}$ CT-related data are not available, but by approximation can be considered close to MRI methodology.

ADPKD, autosomal dominant polycystic kidney disease; N/A, not available; TKV, total kidney volume. 
Fig. 1. A Manual techniques available for estimating total kidney volume in patients with ADPKD. Planimetry method applied to an axial slice on a contrast-enhanced CT image; all cross-sectional slices are manually traced with kidney volume calculated by multiplying all traced areas by slice thickness, and then combining slice volumes (Aa). Stereology applied to a coronal slice using MRI; grid points covering the entire kidney are defined, with the number of intersections within it (black intersections) converted into a pixel count; kidney volume is calculated by summing the products of the resulting areas and the corresponding slice thickness (Ab). Ellipsoid formula applied to a T2-weighted tilted coronal slice $(\mathbf{A c})$ and a sagittal slice $(\mathbf{A d})$; measurements of longitudinal length (L), maximum width (W), and maximal depth (D) are used to calculate renal volume. Mid-slice method applied to a coronal slice using MRI; manual tracing of a contour on a single middle slice image of the kidney is required. After quantification of the area inside the contour ( $\left.\mathrm{A}_{\text {mid_slice }}\right)$, kidney volume $(V)$ is computed by multiplying this area by the number of slices in which the kidney is visible $(n)$, by slice thickness (ST) and by a correction factor (c) experimentally defined [43] (Ae). B Semiautomated renal segmentation procedure, which involves manual selection of reference points (asterisks) in the central slice of the right and left kidney $(\mathbf{B a})$; obtaining rough segmentation based on mean pixel value in adjacent areas (Bb); obtaining kidney contours by reiterating the segmentation procedure using a region-growing algorithm (BC); and final image refinement by applying a curvature motion in regions with negative curvature (Bd).
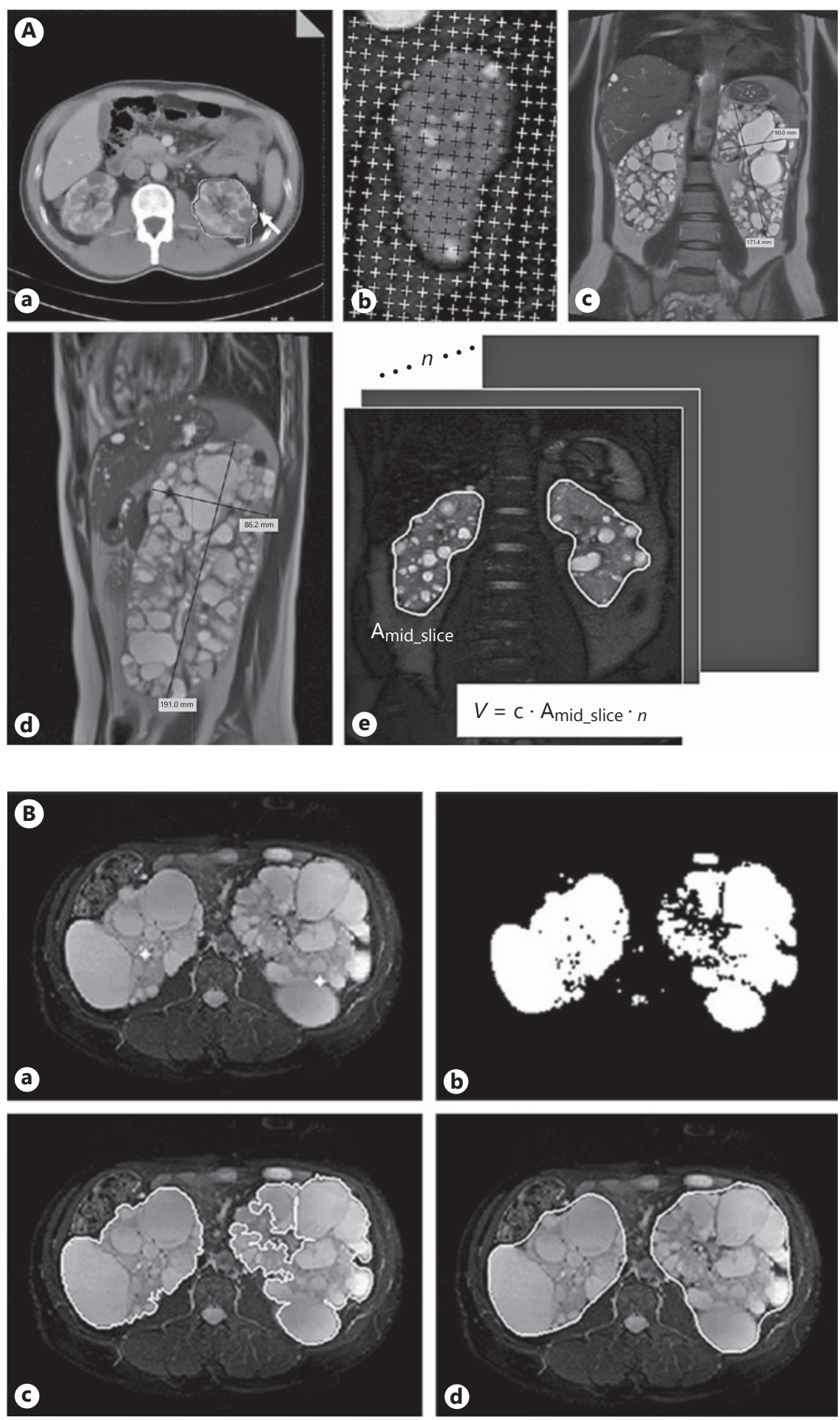

tient selection criteria for treatment with tolvaptan, and in line with data from CRISP, recommended that in the absence of an MRI, patients aged $<45$ years with a kidney length of $>16.5 \mathrm{~cm}$ measured by ultrasound, are likely to have rapid disease progression [14, 24].

Imaging in $\mathrm{ADPKD}$ Progression
Ultrasound has several limitations that can affect the accuracy of TKV estimation and lead to a high variability in measurements. Operator acquisition of incorrect longitudinal and coronal views can result in foreshortening of kidney length, width, and depth and could lead to an

Am J Nephrol 2018;48:67-78 DOI: $10.1159 / 000491022$ 
inaccurate estimate of TKV when applying the ellipsoid equation. In addition, the feasibility of viewing the entire kidney with ultrasound will become more difficult as the disease progresses. Based on previous CRISP data [24], a kidney with a length of $>17 \mathrm{~cm}$ should be considered a poor candidate for ultrasound examination. Furthermore, ultrasound accuracy is largely dependent on the sonographer's expertise; therefore, kidney acquisition should be performed only by professionals specifically trained in the examination of patients with ADPKD, in order to reduce intra- and interobserver variability. Lastly, ensuring adequate patient preparation (e.g., preventing meteorism by abstaining from fiber consumption in the previous $24 \mathrm{~h}$ ) can also help facilitate the acquisition of the kidney and reproducibility of images.

\section{Magnetic Resonance Imaging}

Early CRISP studies used gadolinium (Gd)-enhanced three-dimensional volume-interpolated spoiled gradient-echo coronal $\mathrm{T}_{1}$-weighted scans, with renal volume measured by manual stereology $[15,25,26]$. They showed that MRI could reliably measure kidney volume with minimal bias and low inter- and intraoperator variability. While these media have been associated with nephrogenic systemic fibrosis in patients with kidney disease [27, 28], $T_{2}$-weighted images obtained without contrast medium also allow reliable measurement of renal volumes $[29,30]$. Following CRISP recommendations, and based on our experience, we suggest an MRI acquisition protocol that includes $\mathrm{T}_{2}$-weighted fast spin echo images with fat saturation, in coronal view, with no contrast media injection. We recommend an echo time and repetition time, depending on the imaging system of 3,000-5,000 and 70-200 ms respectively; a field of view of $30-35 \mathrm{~cm}$; a slice thickness of $5 \mathrm{~mm}$; and spacing between slices equal to $0.5 \mathrm{~mm}$.

The ERA-EDTA WGIKD/ERBP statement recommended that MRI be used in clinical practice for identifying ADPKD patients with rapid disease progression [14]. It also suggested that MRI measurements for assessing changes in TKV should be repeated over short periods to minimize intraindividual and intraobserver variability [14], although the availability of this method can be limited and seems to be of little use outside clinical trials.

\section{Computed Tomography}

Concerns over ionizing radiation and nephrotoxic contrast medium limit the usefulness of CT for ongoing assessment and in young patients with $\mathrm{ADPKD}$ who have renal impairment [31]. However, the use of CT is consid- ered an accurate technique for TKV estimation, and compared with MRI, has the advantage of greater availability in clinical centers. Recently, a preliminary study showed the feasibility of using non-contrast-enhanced CT to accurately assess TKV when applying a fully automated estimation method [32]. Utilizing such an approach and considering other rapid-acquisition protocols with low radiation exposure and avoidance of nephrotoxic contrast medium could increase the use of CT for TKV assessment. In addition, although it is recommended that MRI be used instead of CT when considering serial TKV measurements, if a CT scan is already available for a patient, it can be used to accurately calculate TKV.

\section{Measuring Kidney Volume from Imaging Techniques}

\section{Manual Planimetry for MRI or CT}

Manual segmentation involves tracing the kidney outline onto cross-sectional images (Fig. 1Aa). The kidney volume is calculated by multiplying all traced areas by slice thickness, and then combining slice volumes [33, 34]. Previous studies have shown that manual planimetry can allow for highly reproducible and accurate kidney volume measurements $[33,35]$. In line with previous CRISP studies, Kistler et al. [33] applied manual segmentation volumetry to unenhanced MRI scans, and demonstrated high intraobserver and interobserver agreement for volume measurements, with correlation coefficients of 1.000 and 0.996 respectively. The SD of interobserver measurements was small at $\pm 15.687 \mathrm{~cm}^{3}$; less than the change in volume during follow-up at $\pm 25.3 \mathrm{~cm}^{3}$. This method was validated for monitoring changes in early ADPKD within a 6-month interval [33]. While this method offers an accurate estimate of TKV $[33,35]$, the analysis time may be $55 \mathrm{~min}$ [36], limiting its usefulness in clinical practice.

\section{Stereology for MRI or CT}

Stereology requires the definition of specific grid points corresponding to kidney regions in cross-sectional slices covering the entire organ (Fig. 1Ab) [37]. Areas of cysts or renal parenchyma are calculated by counting the number of intersections within them and converting this into a pixel count; renal or cyst volume is calculated by summing the products of the resulting areas and corresponding slice thickness. Chapman et al. [26] showed that this approach yields reliable results for kidney and cyst volumes, with reliability coefficients of 0.998 and 0.961 respectively. A recent comparative study of different kidney volume com- 
putation methods demonstrated that stereology had an accuracy measure that was comparable with planimetry methods, with a percentage error of 6.3 and $2.1 \%$ when using MRI and CT respectively [35]. Although this technique is often considered the gold standard, its accuracy and reliability are influenced by display window settings and grid size [37]. In the research setting, serial imaging of kidney volume using stereology should not be carried out more frequently than every 6 months. However, in clinical practice, this approach is not recommended because of the analysis time required.

\section{Ellipsoidal Formula for Ultrasound, MRI, or CT}

The ellipsoid formula can be used to calculate TKV from manual measurements of length, width, and depth on ultrasound, MRI, or CT scans (Fig. 1Ac, d) [17, 36, 38, 39]. The 2 basic ellipsoid equations are as follows:

$$
\begin{aligned}
& \mathrm{KV}=\pi / 6 \times \mathrm{L} \times \mathrm{W} \times \mathrm{D} \\
& \mathrm{KV}=\pi / 12 \times \mathrm{L} \times(\mathrm{W}+\mathrm{WW}) \times \mathrm{D}
\end{aligned}
$$

where $\mathrm{D}=$ maximum depth; $\mathrm{KV}=$ kidney volume; $\mathrm{L}=$ maximal longitudinal length; $\mathrm{W}=$ maximal width perpendicular to L; and WW = width greater than $\mathrm{W}$.

The maximal width $(\mathrm{W})$ is determined perpendicularly to $\mathrm{L}$ on the same slice that $\mathrm{L}$ is localized on. In addition to $\mathrm{W}$, a greater width than $\mathrm{W}(\mathrm{WW})$ is surveyed on the other tilted coronal slice and is used in MRI and reformatted CT images.

While this approach has been used in long-term studies of the natural history of ADPKD [24, 40], in patients with early ADPKD, the ellipsoid equation lacks the precision to measure small TKV changes [41], which can be due to measuring errors in depth and transverse width of the kidney. It is not recommended to repeat serial imaging more often than every 6 months.

Higashihara et al. [38] showed that intra- and interobserver reproducibility of kidney dimensions and calculated renal volumes derived from MRI scans was good, with an intraclass correlation coefficient of $>0.9$. However, compared with standard planimetry, the ellipsoid formulas used tended to underestimate kidney volume, with the most accurate formula being $\pi / 24 \times \mathrm{L} \times(\mathrm{W}+$ WW $)^{2}$.

Another study found a strong correlation between TKV calculated by the ellipsoid formula and that calculated by stereology $\left(R^{2}=0.979\right)$ - without systematic under- or overestimation of TKV - using the formula $\pi / 6 \times$ $\mathrm{L} \times \mathrm{W} \times \mathrm{D}$ with mean sagittal and coronal length [17] Applying the ellipsoid formula to ultrasound-measured

Imaging in $\mathrm{ADPKD}$ Progression diameters has shown poor intra- and interobserver variation, with a standard deviation of the difference in variation of 21-32 $\mathrm{mL}$, and is not recommended for accurate estimation of TKV [42]. However, it can give a rough estimation that is particularly useful in very mildly affected patients.

\section{Mid-Slice Technique for MRI or CT}

In this technique, the renal volume is calculated from the area of a single middle slice image of the kidney (determined by stereology) multiplied by the number of slices (Fig 1Ae) [43]. The subsequent kidney volumes correlate well with stereology (left kidney, $R^{2}=0.991$; right kidney, $R^{2}=0.994$ ) [43], and have high reproducibility comparable with manual planimetry [36]. However, Sharma et al. [35] showed that, when calculating single kidney volumes with CT, both the mid-slice technique and the ellipsoid formula were significantly less accurate than stereology and manual and semi-automatic planimetry, compared with a reference method of polyline manual tracing. Although significantly faster than manual tracing for calculating kidney volume, this technique is slower than the standard ellipsoid method, taking 15 and 5 min respectively $[35,36]$.

Volume estimates are based on a multiplier linked to the hypothesis that the shape of the kidney is ellipsoidal [43]. This approach therefore relies on geometrical assumptions, and could have a negative impact from the same disadvantages that characterize the ellipsoid formula. With regard to the possible use of this method in other patient populations, the coefficient proposed in the development of this technique is not proven and needs further investigation [43].

\section{Semiautomated Techniques for MRI}

Semiautomated approaches to the calculation of renal and cyst volume have been developed in order to achieve rapid image processing for routine clinical use [44-48]. In one such technique, a single reference point is defined in the central plane of each kidney, and rough segmentation is performed based on the mean value of adjacent pixels. This procedure is reiterated for each slice so as to yield accurate segmentation of TKV (Fig. 1B) [44]. TKVs derived from this technique correlate well with stereology $(R=0.99)$, with good precision (mean percentage error $-0.6 \pm 5.8 \%)$ and low bias $(-5 \mathrm{~mL}, p<0.01)$, making this method applicable to routine clinical practice [30, $49]$. Moreover, there is good correlation between the values obtained with either axial or coronal imaging $\left(R^{2}=\right.$ $0.997)$ and the results obtained using the semiautomated 
approach versus the mid-slice technique $\left(R^{2}=0.990\right)$ [44]. This approach can also be applied to markedly enlarged ADPKD kidneys [44].

\section{Fully Automated Techniques for MRI}

Although semiautomated techniques can overcome many of the limitations of manual techniques, they still require input from experienced users. Fully automated techniques have therefore been developed to analyze MRI scans $[50,51]$, which then need only a final quality check [51].

A spatial prior-probability map of the localization of the kidneys in the abdomen is one example of an automated technique [50]. Another requires baseline segmentation initialization performed by manual tracing of MRI scans acquired during the first visit to allow future segmentation to occur automatically [51]. Both approaches correlate well with manual procedures, with comparable accuracy and reproducibility (spatial prior probability map, $R^{2}=0.97$; baseline segmentation initialization, mean \pm SD Dice similarity coefficient $=$ $0.88 \pm 0.08)[50,51]$. However, manual tracing of a vast set of images from different patients is required to build a reliable spatial probability map on which the accuracy of the automated TKV measurement depends. Reported boundaries of agreement for this technique are approximately $30 \%$, limiting its application in clinical practice [50]. Further drawbacks to using these fully automated techniques include variability in kidney morphology and the fluid composition of the cysts, and the difficulty in discriminating the anatomical boundaries of the kidney [52].

\section{Imaging in Progression Models for ADPKD}

\section{Mayo Clinic Model}

An online tool to calculate the risk of progression using the Mayo Clinic model is available $[17,53]$. The Mayo Clinic model was designed to select patients for clinical trials who might benefit from therapies in development (Fig. 2) [17]. The model can predict eGFR decline and progression to ESRD across various disease stages in patients with 'typical' ADPKD, including patients with early disease and preserved renal function [17].

The model classifies patients according to heightadjusted TKV in relation to age and the subsequent exponential increase in kidney size, with class $1 \mathrm{~A}$ estimated as having a kidney growth rate of $1.5 \%$ per year, and class $1 \mathrm{E}$ having a growth rate of $6 \%$ per year. $\mathrm{Pa}-$ tients in classes $1 \mathrm{C}-1 \mathrm{E}$ are predicted to be at risk of rapid disease progression [17], and tolvaptan treatment has been recommended for these patients, provided their age and eGFR are within appropriate limits [14]. A limitation of the model is that the 95\% CI for predicted eGFR loss and time to ESRD are comparatively wide [17].

The model classifies typical ADPKD presentation (classes $1 \mathrm{~A}-1 \mathrm{E}$ ) as diffuse and bilateral distribution of cysts with equal contribution to TKV, and mild, moderate, or severe replacement of kidney tissue (Fig. 3) [17].

Patients with atypical disease (approximately 10\% overall) mostly show slowly progressing disease $[14,17]$. Atypical presentation of class $2 \mathrm{~A}$ is defined as:

- Unilateral: one normal kidney, with the contralateral kidney experiencing significant renal enlargement caused by diffuse cysts

- Segmental: a pole of one or both kidneys affected by cystic disease, with the remaining renal tissue staying healthy (Fig. 3)

- Asymmetric: significant enlargement of one kidney due to diffuse cystic disease, with minimal diffuse or mild segmental cystic disease in the contralateral kidney

- Lopsided: mild replacement of kidney tissue with atypical cysts in a bilateral distribution.

These class $2 \mathrm{~A}$ patients will mostly be slow progressors.

Atypical presentation of class $2 \mathrm{~B}$ is defined as either bilateral cystic disease with unilateral atrophy and significant renal enlargement or bilateral cystic disease with bilateral atrophy and renal impairment without significant renal enlargement [17]. Class $2 \mathrm{~B}$ patients would only receive a limited benefit from specific therapy.

This model currently represents the best standard for predicting disease progression; however, in the future, it can be expected that a combination of risk markers may provide more accurate predictions.

\section{ADPKD Outcomes Model}

The APDKD outcomes model was developed using TKV and eGFR progression rates derived from the placebo arm of the TEMPO 3:4 trial [54]. The model simulates disease progression of hypothetical patient cohorts over prespecified time horizons, taking into account covariates including age, sex, and baseline TKV. The model showed that differences in baseline TKV can markedly affect time to ESRD, and that younger patient cohorts with large kidneys would enter ESRD at a younger age. For example, the predicted age of progression to ESRD in 


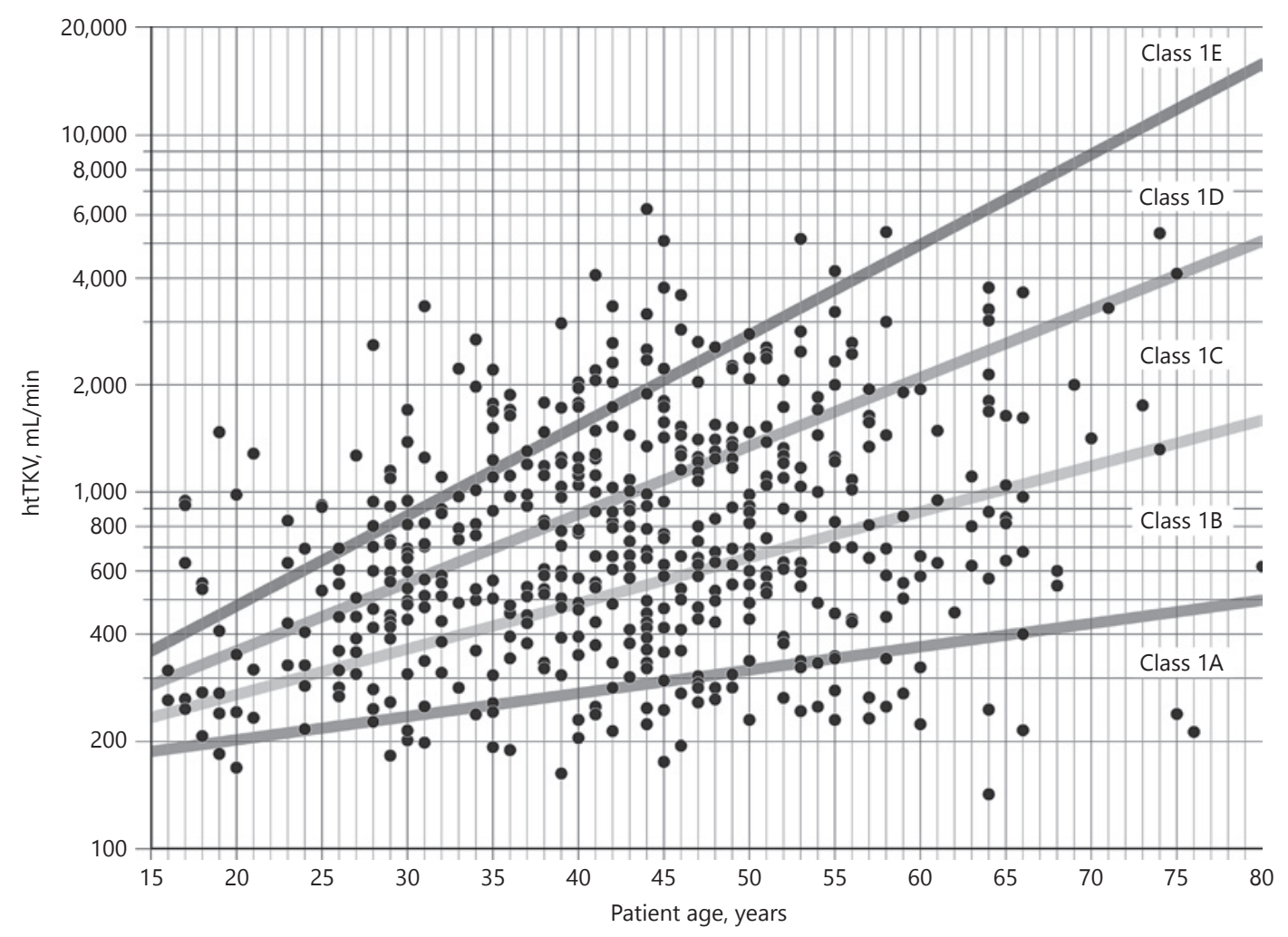

Fig. 2. The Mayo Clinic classification of ADPKD based on heightadjusted TKV (htTKV) and age. Classes A - E have estimated annual kidney growth rates of $<1.5,1.5-3.0,3.0-4.5,4.5-6.0$, and $>6.0 \%$ respectively. Normal values were derived from healthy kidney donors. Republished with permission of the American Society of Nephrology, from Imaging classification of ADPKD: a simple model for selecting patients for clinical trials, Irazabal et al. [17].
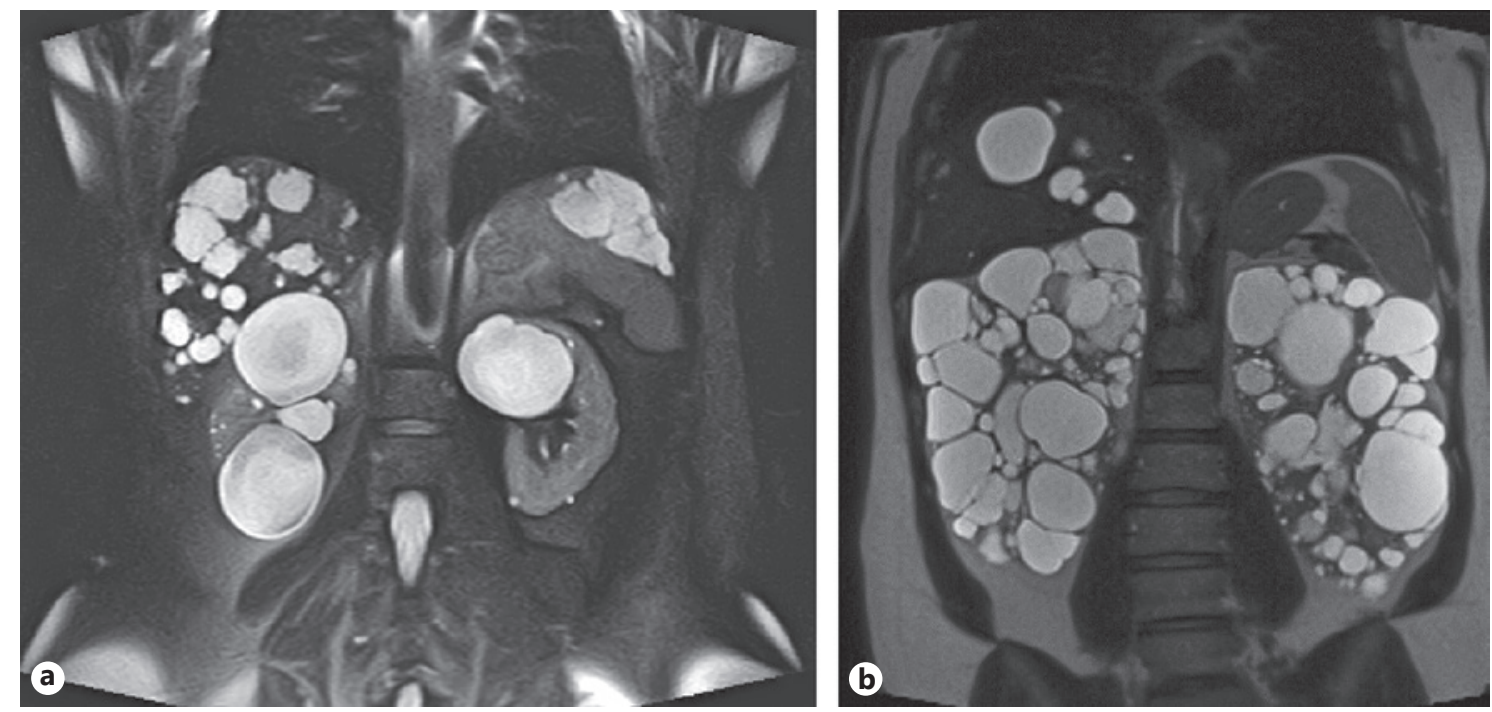

Fig. 3. Mayo Clinic model example of atypical ADPKD presentation, lopsided type (mild kidney tissue replacement, in which 5 cysts account for $>50 \%$ of total kidney volume (TKV). In the magnetic resonance slice shown, only 4 cysts are visible; the fifth stands in another plane of slice; right kidney volume, $325 \mathrm{~mL}$; left kidney volume, $308 \mathrm{~mL}$; TKV, $661 \mathrm{~mL}$; cysts volume, $358 \mathrm{~mL}$; (a) and typical ADPKD presentation (b). 
a patient aged 30 with eGFR $110 \mathrm{~mL} / \mathrm{min} / 1.73 \mathrm{~m}^{2}$ and a TKV of 1,000 mL, would be 49-54 years, compared with 60-65 years for a patient aged 45 years with eGFR $80 \mathrm{~mL} /$ $\mathrm{min} / 1.73 \mathrm{~m}^{2}$ and a TKV of $1,000 \mathrm{~mL}$ [54].

\section{Conclusions}

Classifying patients with ADPKD into a progression risk category requires an accurate and expansive approach. The level of accuracy required is determined by that accepted and required according to local guidelines. If the clinical question is whether to initiate long-term treatment, the clinical history of the patient, tolerability profile of the drug, and financial cost - alongside a high degree of certainty of the progression category - is needed to inform the decision.

Although MRI and CT scans are recommended in the majority of guidelines, ultrasound could be used for initial screening and may be sufficient in the case of nearnormal estimated TKV in cases in which treatment is not indicated. In addition, ultrasound may be useful for identifying young patients with ADPKD who have clearly enlarged kidney volumes for their age and height, although MRI is still recommended.

For clinical questions requiring a low degree of accuracy, ultrasound is considered sufficient and most appropriate in clinical practice. Generally, however, in patients with even mildly enlarged kidneys, the error associated with the Mayo Clinic model classification based on ultrasound is high, and there is an unacceptable risk of misclassifying patients into an inappropriate risk category. We therefore suggest using a protocol that delimits candidates prior to performing these imaging techniques (preferably MRI) so as to obtain an accurate measure- ment of TKV. We suggest using an accurate protocol, predominantly in patients with TKV calculated by ultrasound using the ellipsoid formula in Mayo Clinic classes $1 \mathrm{~B}-1 \mathrm{C}$. Patients classed by ultrasound as $1 \mathrm{~A}, 1 \mathrm{D}$, or $1 \mathrm{E}$ are unlikely to change classes if measurements are performed using more accurate techniques.

All the imaging techniques discussed can play a role in evaluating TKV in ADPKD, alongside consideration of other factors including genotype and age. In the ideal clinical scenario, an accurate MRI-based risk stratification approach is appropriate and could be economically sustainable.

\section{Acknowledgments}

The authors would like to thank James Wallis, MRes of Cello Health MedErgy, who, under the direction of the authors, produced the first draft of this manuscript and provided editorial assistance throughout the manuscript development process. The editorial services provided by Cello Health MedErgy were funded by Otsuka Pharmaceutical Europe Ltd., in accordance with Good Publication Practice (GPP3) guidelines (http://www.ismpp.org/gpp3).

\section{Disclosure Statement}

The authors of this manuscript have the following disclosures: R.M. has received personal fees from Otsuka Pharmaceutical Italia for advisory board membership outside of the submitted work; T.M. has given lectures for Otsuka; R.T. has given lectures for Otsuka and has previously belonged to the Independent Data Monitoring Committee of the TEMPO trial; C.C. has none to declare with regard to this manuscript. Editorial support for the development of this manuscript was funded by Otsuka Pharmaceutical Europe Ltd.

The results presented in this paper have not previously been published elsewhere in whole or in part.

\section{References}

1 Spithoven EM, Kramer A, Meijer E, Orskov B, Wanner C, Abad JM, et al: Renal replacement therapy for autosomal dominant polycystic kidney disease (ADPKD) in Europe: prevalence and survival - an analysis of data from the ERA-EDTA Registry. Nephrol Dial Transplant 2014;29:iv15-iv25.

2 Grantham JJ, Geiser JL, Evan AP: Cyst formation and growth in autosomal dominant polycystic kidney disease. Kidney Int 1987; 31:1145-1152.

3 Grantham JJ: Autosomal dominant polycystic kidney disease. N Engl J Med 2008;359:14771485.
4 Grantham JJ, Chapman AB, Torres VE: Volume progression in autosomal dominant polycystic kidney disease: the major factor determining clinical outcomes. Clin J Am Soc Nephrol 2006;1:148-157.

5 Rossetti S, Consugar MB, Chapman AB, Torres VE, Guay-Woodford LM, Grantham JJ, et al: Comprehensive molecular diagnostics in autosomal dominant polycystic kidney disease. J Am Soc Nephrol 2007; 18:2143-2160.

6 Reed B, McFann K, Kimberling WJ, Pei Y, Gabow PA, Christopher K, et al: Presence of de novo mutations in autosomal dominant polycystic kidney disease patients without fami- ly history. Am J Kidney Dis 2008;52:10421050 .

7 Cornec-Le Gall E, Audrézet MP, Chen JM, Hourmant M, Morin MP, Perrichot R, et al: Type of PKD1 mutation influences renal outcome in ADPKD. J Am Soc Nephrol 2013; 24:1006-1013.

8 Chapman AB, Devuyst O, Eckardt KU, Gansevoort RT, Harris T, Horie S, et al: Autosomal-dominant polycystic kidney disease (ADPKD): executive summary from a Kidney Disease: Improving Global Outcomes (KDIGO) Controversies Conference. Kidney Int 2015;88:17-27. 
9 Torres VE, Chapman AB, Devuyst O, Gansevoort RT, Grantham JJ, Higashihara E, et al: Tolvaptan in patients with autosomal dominant polycystic kidney disease. N Engl J Med 2012;367:2407-2418.

10 European Medicines Agency: Public Assessment Report Jinarc 2015. Available from: http://www.ema.europa.eu/docs/en_GB/ document_library/EPAR_-_Public_assessment_report/human/002788/WC500187923. pdf (accessed November 29, 2017).

11 Food and Drug Admistration: NDA approval 2018. https://www.accessdata.fda.gov/drugsatfda_docs/appletter/2018/204441Orig1s00 0ltr.pdf (accessed June 13, 2017).

12 Schrier RW, Abebe KZ, Perrone RD, Torres VE, Braun WE, Steinman TI, et al: Blood pressure in early autosomal dominant polycystic kidney disease. N Engl J Med 2014;371:22552266.

13 Grantham JJ, Cook LT, Torres VE, Bost JE, Chapman AB, Harris PC, et al: Determinants of renal volume in autosomal-dominant polycystic kidney disease. Kidney Int 2008;73:108-116.

14 Gansevoort RT, Arici M, Benzing T, Birn H, Capasso G, Covic A, et al: Recommendations for the use of tolvaptan in autosomal dominant polycystic kidney disease: a position statement on behalf of the ERA-EDTA Working Groups on Inherited Kidney Disorders and European Renal Best Practice. J Am Soc Nephrol 2016;31:337-348.

15 Grantham JJ, Torres VE, Chapman AB, Guay-Woodford LM, Bae KT, King BF, et al: Volume progression in polycystic kidney disease. N Engl J Med 2006;354:2122-2130.

16 Chapman AB, Bost JE, Torres VE, GuayWoodford L, Bae KT, Landsittel D, et al: Kidney volume and functional outcomes in autosomal dominant polycystic kidney disease Clin J Am Soc Nephrol 2012;7:479-486.

17 Irazabal MV, Rangel LJ, Bergstralh EJ, Osborn SL, Harmon AJ, Sundsbak JL, et al: Imaging classification of autosomal dominant polycystic kidney disease: a simple model for selecting patients for clinical trials. J Am Soc Nephrol 2015;26:160-172.

18 Grantham JJ, Torres VE: The importance of total kidney volume in evaluating progression of polycystic kidney disease. Nat Publ Gr 2016;12:667-677.

19 Perico N, Antiga L, Caroli A, Ruggenenti P, Fasolini G, Cafaro M, et al: Sirolimus therapy to halt the progression of ADPKD. J Am Soc Nephrol 2010;21:1031-1040.

20 Torres VE, Chapman AB, Devuyst O, Gansevoort RT, Perrone RD, Dandurand A, et al: Multicenter, open-label, extension trial to evaluate the long-term efficacy and safety of early versus delayed treatment with tolvaptan in autosomal dominant polycystic kidney disease: the TEMPO 4:4 Trial. Nephrol Dial Transplant 2017;33:447-489.

21 Perrone RD, Neville J, Chapman AB, Gitomer BY, Miskulin DC, Torres VE, et al: Therapeutic area data standards for autosomal domi- nant polycystic kidney disease: a report from the Polycystic Kidney Disease Outcomes Consortium (PKDOC). Am J Kidney Dis 2015;66:583-590.

22 Perrone RD, Mouksassi MS, Romero K, Czerwiec FS, Chapman AB, Gitomer BY, et al: Total kidney volume is a prognostic biomarker of renal function decline and progression to end-stage renal disease in patients with autosomal dominant polycystic kidney disease. Kidney Int Rep 2017;2:442-450.

23 Yu ASL, Shen C, Landsittel DP, Harris PC, Torres VE, Mrug M, et al: Baseline total kidney volume and the rate of kidney growth are associated with chronic kidney disease progression in Autosomal Dominant Polycystic Kidney Disease. Kidney Int 2018;93:753-760.

24 Bhutani H, Smith V, Rahbari-Oskoui F, Mittal A, Grantham JJ, Torres VE, et al: A comparison of ultrasound and magnetic resonance imaging shows that kidney length predicts chronic kidney disease in autosomal dominant polycystic kidney disease. Kidney Int 2015;88:146-151.

25 Bae KT, Tao C, Zhu F, Bost JE, Chapman AB, Grantham JJ, et al: MRI-based kidney volume measurements in ADPKD: reliability and effect of gadolinium enhancement. Clin J Am Soc Nephrol 2009;4:719-725.

26 Chapman AB, Guay-Woodford LM, Grantham JJ, Torres VE, Bae KT, Baumgarten DA, et al: Renal structure in early autosomal-dominant polycystic kidney disease (ADPKD): the Consortium for Radiologic Imaging Studies of Polycystic Kidney Disease (CRISP) cohort. Kidney Int 2003;64:1035-1045.

27 High WA, Ayers RA, Chandler J, Zito G, Cowper SE: Gadolinium is detectable within the tissue of patients with nephrogenic systemic fibrosis. J Am Acad Dermatol 2007; 56:21-26.

28 Thakral C, Abraham JL: Gadolinium-induced nephrogenic systemic fibrosis is associated with insoluble Gd deposits in tissues: in vivo transmetallation confirmed by microanalysis. J Cutan Pathol 2009;36:1244-1254.

29 Lee YR, Lee KB: Reliability of magnetic resonance imaging for measuring the volumetric indices in autosomal-dominant polycystic kidney disease: correlation with hypertension and renal function. Nephron Clin Pract 2006; 103:c173-c180.

30 Mignani R, Corsi C, De Marco M, Caiani EG Santucci G, Cavagna E, et al: Assessment of kidney volume in polycystic kidney disease using magnetic resonance imaging without contrast medium. Am J Nephrol 2011;33:176184.

31 Mai J, Lee VW, Lopez-Vargas P, Vladica P, Rangan GK: KHA-CARI autosomal dominant polycystic kidney disease guideline: monitoring disease progression. Semin Nephrol 2015; 35:565-571.

32 Turco D, Valinoti M, Martin EM, Tagliaferri C, Scolari F, Corsi C: Fully automated segmentation of polycystic kidneys from noncontrast computed tomography. A feasibility study and preliminary results. Acad Radiol 2018;31-32.

33 Kistler AD, Poster D, Krauer F, Weishaupt D, Raina S, Senn O, et al: Increases in kidney volume in autosomal dominant polycystic kidney disease can be detected within 6 months. Kidney Int 2009;75:235-241.

34 King BF, Reed JE, Bergstralh EJ, Sheedy PF 2nd, Torres VE: Quantification and longitudinal trends of kidney, renal cyst, and renal parenchyma volumes in autosomal dominant polycystic kidney disease. J Am Soc Nephrol 2000;11:1505-1511.

35 Sharma K, Caroli A, Quach LV, Petzold K, Bozzetto M, Serra AL, et al: Kidney volume measurement methods for clinical studies on autosomal dominant polycystic kidney disease. PLoS One 2017;12:e0178488.

36 Spithoven EM, van Gastel MD, Messchendorp AL, Casteleijn NF, Drenth JP, Gaillard $\mathrm{CA}$, et al: Estimation of total kidney volume in autosomal dominant polycystic kidney disease. Am J Kidney Dis 2015;66:792-801.

37 Bae KT, Commean PK, Lee J: Volumetric measurement of renal cysts and parenchyma using MRI: phantoms and patients with polycystic kidney disease. J Comput Assist Tomogr 2000;24:614-619.

38 Higashihara E, Nutahara K, Okegawa T, Tanbo M, Hara H, Miyazaki I, et al: Kidney volume estimations with ellipsoid equations by magnetic resonance imaging in autosomal dominant polycystic kidney disease. Nephron 2015; 129:253-262.

39 O'Neill WC, Robbin ML, Bae KT, Grantham JJ, Chapman AB, Guay-Woodford LM, et al: Sonographic assessment of the severity and progression of autosomal dominant polycystic kidney disease: the Consortium of Renal Imaging Studies in Polycystic Kidney Disease (CRISP). Am J Kidney Dis 2005;46:1058-1064.

40 Gabow PA, Johnson AM, Kaehny WD, Kimberling WJ, Lezotte DC, Duley IT, et al: Factors affecting the progression of renal disease in autosomal-dominant polycystic kidney disease. Kidney Int 1992;41:1311-1319.

41 Hammoud S, Tissier AM, Elie C, Pousset M, Knebelman B, Joly D, et al: Ultrasonographic renal volume measurements in early autosomal dominant polycystic disease: comparison with CT-scan renal volume calculations. Diagn Interv Imaging 2015;96:65-71.

42 Bakker J, Olree M, Kaatee R, de Lange EE, Moons KG, Beutler JJ, et al: Renal volume measurements: accuracy and repeatability of US compared with that of MR imaging. Radiology 1999;211:623-628.

43 Bae KT, Tao C, Wang J, Kaya D, Wu Z, Bae JT, et al: Novel approach to estimate kidney and cyst volumes using mid-slice magnetic resonance images in polycystic kidney disease. Am J Nephrol 2013;38:333-341.

44 Turco D, Severi S, Mignani R, Aiello V, Magistroni R, Corsi C: Reliability of total renal volume computation in polycystic kidney disease from magnetic resonance imaging. Acad Radiol 2015;22:1376-1384. 
45 Bae K, Park B, Sun H, Wang J, Tao C, Chapman $A B$, et al: Segmentation of individual renal cysts from MR images in patients with autosomal dominant polycystic kidney disease. Clin J Am Soc Nephrol 2013;8:10891097.

46 Racimora D, Vivier $\mathrm{PH}$, Chandarana $\mathrm{H}$, Rusinek H: Segmentation of polycystic kidneys from MR images. In: Karssemeijer N, Summers RM (eds): Med Imaging. 2010, vol 7624, pp 540-550.

47 Cohen BA, Barash I, Kim DC, Sanger MD, Babb JS, Chandarana H: Intraobserver and interobserver variability of renal volume measurements in polycystic kidney disease using a semiautomated MR segmentation algorithm. Am J Roentgenol 2012;199:387-393.

48 Kline TL, Edwards ME, Korfiatis P, Akkus Z, Torres VE, Erickson BJ: Semiautomated segmentation of polycystic kidneys in T2weighted MR images. Am J Roentgenol 2016; 207:605-613.

49 Corsi C, Mignani R, Turco D, Magistroni R, Severi S: Comment on the paper: "novel ap- proach to estimate kidney and cyst volumes using mid-slice magnetic resonance images in polycystic kidney disease." Am J Nephrol 2014;39:163-164.

50 Kim Y, Ge Y, Tao C, Zhu J, Chapman AB, Torres VE, et al: Automated segmentation of kidneys from MR images in patients with autosomal dominant polycystic kidney disease. Clin J Am Soc Nephrol 2016;11:576-584.

51 Kline TL, Korfiatis P, Edwards ME, Warner JD, Irazabal MV, King BF, et al: Automatic total kidney volume measurement on followup magnetic resonance images to facilitate monitoring of autosomal dominant polycystic kidney disease progression. Nephrol Dial Transplant 2016;31:241-248.

52 Bae KT, Grantham JJ: Imaging for the prognosis of autosomal dominant polycystic kidney disease. Nat Rev Nephrol 2010;6:96-106.

53 Mayo Foundation and Medical Education and Research: Imaging classification of ADPKD: A simple model for selecting patients for clinical trials. Mayo ADPKD class calculator. Available from: http://www.mayo.edu/research/ documents/pkd-center-adpkd-classification/ doc-20094754 (accessed November 29, 2017).

54 McEwan P, Bennett Wilton H, Ong ACM, Ørskov B, Sandford R, Scolari F, et al: A model to predict disease progression in patients with autosomal dominant polycystic kidney disease (ADPKD): the ADPKD Outcomes Model. BMC Nephrol 2018;37.

55 Ars E, Bernis C, Fraga G, Martinez V, Martins J, Ortiz A, et al: Spanish guidelines for the management of autosomal dominant polycystic kidney disease. Nephrol Dial Transplant 2014;29(suppl 4):95-105.

56 Sise C, Kusaka M, Wetzel LH, Winklhofer F, Cowley BD, Cook LT, et al: Volumetric determination of progression in autosomal dominant polycystic kidney disease by computed tomography. Kidney Int 2000;58:2492-2501.

57 Turco D, Busutti M, Mignani R, Magistroni $\mathrm{R}$, Corsi C: Comparison of total kidney volume quantification methods in autosomal dominant polycystic disease for a comprehensive disease assessment. Am J Nephrol 2017;373-379. 